\title{
Geometric Measurement of Topological Susceptibility on Large Latticest
}

\author{
J. Grandy and R. Gupta ${ }^{a}$

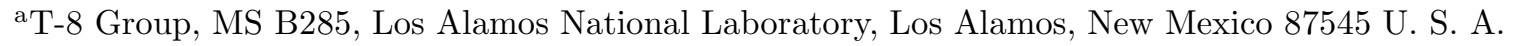

The topological susceptibility of the quenched QCD vacuum is measured on large lattices for three $\beta$ values from 6.0 to 6.4. Charges possibly induced by $O(a)$ dislocations are identified and shown to have little effect on the measured susceptibility. As $\beta$ increases, fewer such questionable charges are found. Scaling is checked by

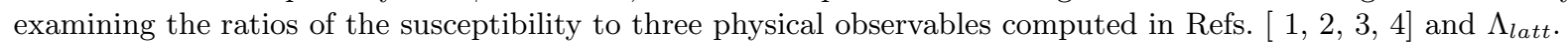

The topological susceptibility of the QCD vacuum is defined by

$$
\chi_{t}=\left\langle Q^{2}\right\rangle / V, \quad Q=\frac{-1}{16 \pi^{2}} \int_{V} d^{4} x F(x) \tilde{F}(x) .
$$

The susceptibility is related to the $\eta^{\prime}$ and $\eta$ masses in the chiral large- $N_{c}$ limit through the WittenVeneziano formula[ 5]

$\chi_{t}=\frac{f_{\pi}^{2}}{6}\left(m_{\eta^{\prime}}^{2}+m_{\eta}^{2}-2 m_{K}^{2}\right)=(180 M e V)^{4}$.

The computation of $\chi_{t}$ on a lattice is not straightforward. The simplest method, replacing $F \tilde{F}$ by a product of plaquettes, suffers mixing with $F^{2}$ and a constant[ [G], and a large perturbatively determined renormalization factor, but cooling and smearing algorithms have been proposed to refine this method[ 7, 8]. The geometric method, first proposed by Lüscher[ [9], is not susceptible to multiplicative renormalization but contains additive divergences from compact dislocations[10] so that

$$
\chi_{t}^{\text {measured }}=\chi_{t}+\int_{p_{0}}^{\infty} d p C(p) a^{p} .
$$

The dislocation described by Göckeler et al. [11] produces a power $p_{0}<0$ in (3) and thus a power law divergence in the measured susceptibility as $a \rightarrow 0$. The factor $C(p)$ for $p<0$ is crucial to determining the onset of divergent behavior in the

\footnotetext{
*Presented by J. Grandy. Work supported by University of California under DOE Contract W-7405-ENG-36. Computer time on C90 provided by NERSC and PSC Supercomputing Centers.
}

measured susceptibility for finite $a$, and we investigate whether divergent terms affect the result at realistic values of $a$ used in our calculation.

We are examining several aspects of topology on the lattice. First, we compute $\chi_{t}$ using the geometric method and examine the systematic effect of dislocations in the range $\beta=6.0$ to $\beta=6.4$. We plan to study the various methods of measuring $\chi_{t}$, including cooling, and use the geometric topological charge density to examine the spin asymmetry in deep inelastic scattering off a proton. At present we have completed the first stage and report the results here.

Our lattice ensembles are listed in Table 1. We evaluate the integrals derived by Göckeler et al.[ 12. on the surfaces of each hypercube on the lattice, obtaining a local integer for each hypercube, and add the local integers in each configuration to obtain the topological charge $Q$. The integration requires a two-stage process. First, all hypercubes are integrated using two different coarse $\left(\sim 5^{3}\right)$ cubic meshes. Hypercubes which show no definite evidence of convergence toward zero are integrated more accurately, with $\sim 10^{3}$ meshes on subcubes. Most hypercubes are brought within $10^{-4}$ to $10^{-5}$ of an integer with the finer mesh, but a few difficult hypercubes require further refinement. The number of difficult hypercubes per unit topological charge decreases as $\beta$ increases.

We attempt to separate spurious charges, caused by low-action dislocations, from physical charges. These dislocations in $S U(2)$ typically contain a central plaquette near -1 and have a radius of about 1 lattice spacing[ 11]. Since the topological structure of $S U(3)$ is determined by 


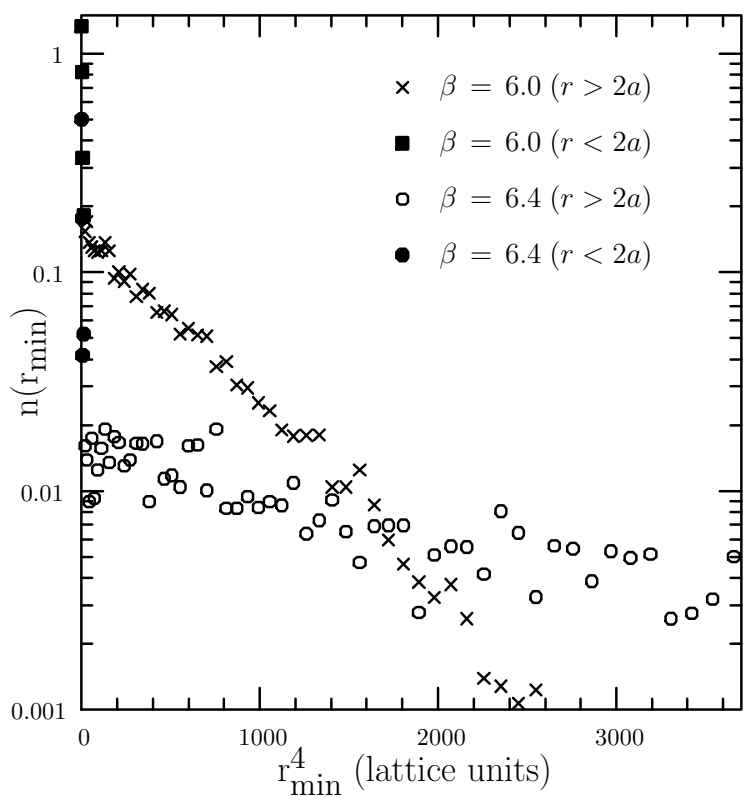

Figure 1. Normalized nearest neighbor correlations from charged hypercubes to nearest plaque-

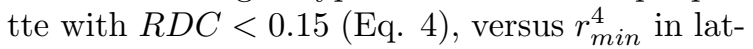
tice units. Highlighted correlations at $r_{\min }<2 a$ indicate possible compact dislocations.

embedded $S U(2)$ windings, we search for correlations between hypercubes of nonzero charge and plaquettes near the $S U(3)$ cut locus, the analog to the -1 group element in $S U(2)$. Plaquettes whose radial distance to cut locus (RDC)[ 13],

$R D C=\left(2 \sum_{i=1}^{3} \theta_{i}^{2}\right)^{1 / 2} \quad\left(\frac{1}{\theta_{3}-\theta_{1}}-\frac{1}{2 \pi}\right)$

is less than 0.15 are selected for presentation here. In this definition (4) the $\theta_{i}$ are the three eigenangles of the $S U(3)$ matrix in ascending order, so that their sum is zero. For each charged hypercube in the ensemble the distance $r_{\min }$ from the center of the hypercube to the center of the nearest selected plaquette is found. The number of occurrences at each lattice distance $r_{\text {min }}, N\left(r_{\text {min }}\right)$ is divided by the weight factor $g\left(r_{\min }\right)$, the number of available plaquettes at distance $r_{\min }$, to obtain a normalized nearest neighbor correlation

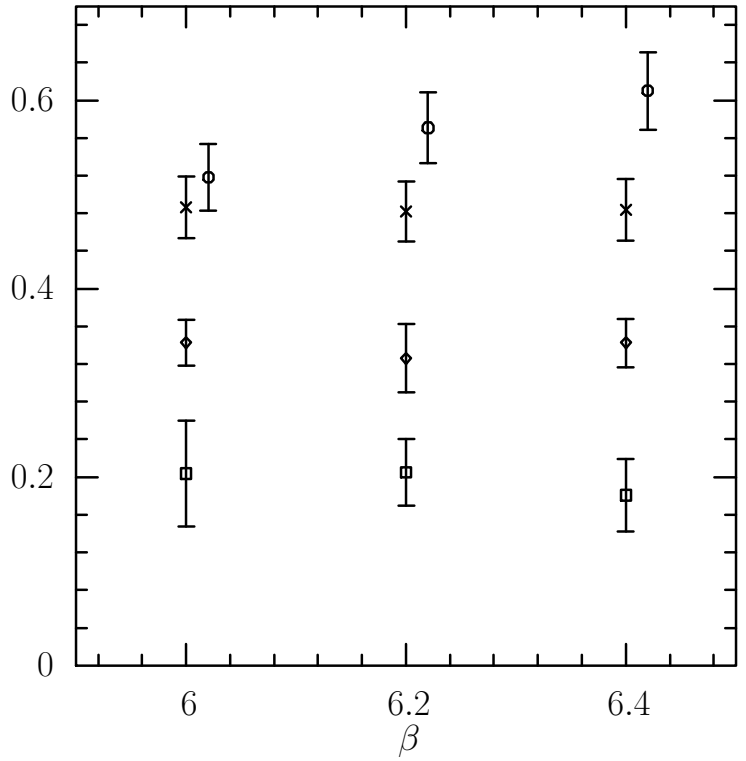

Figure 2. $\chi_{t}^{1 / 4} / \sqrt{\sigma}$ (circles), $\chi_{t}^{1 / 4} /\left(100 \Lambda_{\text {latt }}\right)$ (crosses), $\chi_{t}^{1 / 4} / m_{\rho}$ (diamonds), and $\chi_{t}^{1 / 4} /\left(10 f_{\pi}\right)$ (squares) versus $\beta$. Scaling is indicated when the ratios are independent of $\beta$. The actual values of these ratios are unimportant, since we consider only their dependence on $\beta$. The $16^{3} \times 40$ lattice results are shown for $\beta=6.0$.

$n\left(r_{\min }\right)$. If the charges and nearly critical plaquettes are decoupled, $n\left(r_{\min }\right)$ will fall exponentially as a function of volume spanned by $r_{m i n}$, $n\left(r_{\min }\right) \sim e^{-\alpha r_{\text {min }}^{4}}$.

As we investigate possible dislocations, we find some encouraging results. Our plot (Fig. 11) shows $n\left(r_{\min }\right)$ decaying exponentially as a function of $r_{\text {min }}^{4}$, except for a dramatic rise for $r_{\min }<$ $2 a$, indicating that questionable charges within $2 a$ of a nearly critical plaquette may indeed be dominated by compact dislocations which are lattice artifacts. Where $N_{q}$ is the number of questionable charges in a configuration, we find that $\left\langle N_{q}\right\rangle /\left\langle Q^{2}\right\rangle=0.21$ at $\beta=6.0$, and 0.05 at $\beta=6.4$. This decrease suggests that for the $\beta$ values considered here the additive term in Eq. 3 is dominated by the $p>0$ part of the integral, and 
Table 1

Lattice Ensembles and Results

\begin{tabular}{ccccc}
\hline$\beta$ & $\begin{array}{c}\text { Lattice } \\
\text { Size }\end{array}$ & $\begin{array}{c}\text { Sample } \\
\text { Size }\end{array}$ & $\begin{array}{c}\chi_{t}^{1 / 4}(\sqrt{\sigma}) \\
(\mathrm{MeV})\end{array}$ & $\begin{array}{c}\chi_{t}^{1 / 4}\left(m_{\rho}\right) \\
(\mathrm{MeV})\end{array}$ \\
\hline 6.0 & $16^{3} \times 40$ & 34 & $228(15)$ & $264(19)$ \\
6.0 & $24^{3} \times 40$ & 23 & $220(29)$ & $255(34)$ \\
6.2 & $32^{3} \times 48$ & 22 & $251(16)$ & $251(28)$ \\
6.4 & $32^{3} \times 48$ & 21 & $268(18)$ & $264(20)$ \\
\hline
\end{tabular}

the divergent part $p<0$ gives at most a negligible contribution to our measured $\chi_{t}$. Also, as $\beta$ increases, the physical charge is spread over a larger lattice volume and the short range peak of $n\left(r_{\text {min }}\right)$ becomes more pronounced relative to the background exponential, allowing a cleaner filtering of dislocation-induced charges. Upon removing these charges we find that $\chi_{t}$ changes by about $1 / 3$ of the statistical uncertainty for $\beta=6.0$ and only about $1 / 10$ of the uncertainty for $\beta=6.4$. We take this as evidence that low-action dislocations have little effect on our measured susceptibility, and present results without removing questionable charges. The decrease of $\left\langle N_{q}\right\rangle /\left\langle Q^{2}\right\rangle$ and the small effect of questionable charges on measured $\chi_{t}$ are also observed for other values of the $R D C$ cutoff. A detailed analysis of the $R D C$ cutoff will be presented in a forthcoming paper[14].

Since our calculation of $\chi_{t}$ spans a wide range of $\beta$ we can check for scaling by plotting dimensionless ratios of $\chi_{t}^{1 / 4}$ to four other quantities versus $\beta$. Our computed values for $\chi_{t}^{1 / 4} a$ are $0.114(8)$ at $\beta=6.0$ (16 $6^{3}$ lattice), $0.110(14)$ at $\beta=6.0$ (24 $4^{3}$ lattice), $0.090(6)$ at $\beta=6.2$, and $0.072(5)$ at $\beta=6.4$. The height of each set of points is irrelevant since our concern is whether the ratios are independent of $\beta$. Scaling with $m_{\rho}, f_{\pi}$, and $\Lambda_{\text {latt }}$ derived from the two-loop

Table 2

Lattice Observables (Lattice Units)

\begin{tabular}{|c|c|c|c|}
\hline $\bar{\beta}$ & $\sigma^{1 / 2} a$ & $m_{\rho} a$ & $f_{\pi} a$ \\
\hline$\overline{6.0}$ & $0.220(2)[$ & $0.333(8)[3]$ & $0.056(15)[3]$ \\
\hline 6.2 & $0.158(1)[$ & $0.277(25)[2]$ & $0.044(7)[2]$ \\
\hline 6.4 & $0.119(2)[$ & $0.211(7)[4]$ & $0.040(8)[$ \\
\hline
\end{tabular}

formula is apparent, but a possible slope exists in $\chi_{t}^{1 / 4} / \sqrt{\sigma}$. We present physical values derived from $m_{\rho}=770 \mathrm{MeV}$ and $\sqrt{\sigma}=440 \mathrm{MeV}$ in Table 1. Although the issue of scaling still must be resolved, we see that these results are consistent with earlier results by Göckeler et al. [12], computed on much smaller lattices. Consistency between the smaller and larger lattices at $\beta=6.0$ indicates that our lattices are large enough to control finite size effects. We conclude that the geometric method yields $\chi_{t}$ about a factor of four higher than both the Witten-Veneziano prediction and the $\chi_{t} \approx(180 \mathrm{MeV})^{4}$ obtained by cool$\operatorname{ing}$ [ 7], and that the superficially divergent part of Eq. (3) is unlikely to cause this discrepancy.

\section{REFERENCES}

[1] Schilling, K. and G. S. Bali, PRINT-93-0666 (Wuppertal), August 1993.

[2] C.R. Allton, et al. (The UKQCD Collaboration), EDINBURGH-92-507, June 1993.

[3] Cabasino, S. et al. (The APE Collaboration), Phys. Lett. B258, 195 (1991).

[4] Abada, A., et al., Nucl. Phys. B376, 172 (1992).

[5] Di Vecchia, P., F. Nicodemi, R. Pettorino, and G. Veneziano, Nucl. Phys. B181, 318 (1981).

[6] Campostrini, M., A. Di Giacomo, H. Panagopoulos, and E. Vicari, Nucl. Phys. B329, 683 (1990).

[7] Teper, M., Phys. Lett. B202, 553 (1988).

[8] Teper, M., Phys. Lett. B232, 227 (1989).

[9] Lüscher, M., Commun. Math. Phys. 85, 39 (1982).

[10] Kremer, M., A. S. Kronfeld, M. L. Laursen, C. Schierholz, C. Schleiermacher, U. J. Wiese, Nucl. Phys. B305, 109 (1988).

[11] Göckeler, M., A. S. Kronfeld, M. L. Laursen, G. Schierholz, and U. -J. Wiese, Phys. Lett. B233, 192 (1989).

[12] Göckeler, M., A. S. Kronfeld, M. L. Laursen, G. Schierholz, and U. -J. Wiese, Nucl. Phys. B292, 349 (1987).

[13] Lasher, G., Nucl. Phys. B (Proc. Suppl.) 9, 416 (1989).

[14] Grandy, J., and R. Gupta (in progress). 